\title{
Floor Sensors of Animal Weight and Gait for Precision Livestock Farming
}

DOI:

10.1109/ICSENS.2017.8234202

Document Version

Final published version

Link to publication record in Manchester Research Explorer

\section{Citation for published version (APA):}

Vaughan, J., Green, P., Salter, M., Grieve, B., \& Ozanyan, K. (2017). Floor Sensors of Animal Weight and Gait for Precision Livestock Farming. 978-980. Paper presented at IEEE Sensors 2017 Conference, Glasgow, United Kingdom. https://doi.org/10.1109/ICSENS.2017.8234202

\section{Citing this paper}

Please note that where the full-text provided on Manchester Research Explorer is the Author Accepted Manuscript or Proof version this may differ from the final Published version. If citing, it is advised that you check and use the publisher's definitive version.

\section{General rights}

Copyright and moral rights for the publications made accessible in the Research Explorer are retained by the authors and/or other copyright owners and it is a condition of accessing publications that users recognise and abide by the legal requirements associated with these rights.

\section{Takedown policy}

If you believe that this document breaches copyright please refer to the University of Manchester's Takedown Procedures [http://man.ac.uk/04Y6Bo] or contact uml.scholarlycommunications@manchester.ac.uk providing relevant details, so we can investigate your claim.

\section{OPEN ACCESS}




\title{
Floor Sensors of Animal Weight and Gait for Precision Livestock Farming
}

\author{
John Vaughan, Peter M Green, Michael Salter ${ }^{1}$, Bruce Grieve and Krikor B Ozanyan \\ School of Electrical and Electronic Engineering, The University of Manchester \\ Sackville str., Manchester M13 9PL, United Kingdom \\ ${ }^{1}$ AB Agri Ltd, 64 Innovation Way, Lynch Wood, Peterborough, PE2 6FL, United Kingdom \\ Corresponding author: <Bruce.Grieve@Manchester.ac.UK>
}

\begin{abstract}
We report first results on developing smart sensor systems for the automatic and frequent collection of animal weight and gait data, under the hostile conditions of a livestock farm. The novelty in our approach is to sense frequently the animals' floor contact, in suitably chosen locations, under natural and unobtrusive conditions. We demonstrate a pilot low profile rubberized mat sensor heads, delivering a large number of plastic optical fiber transmission measurements taken frequently from individual deformation sensors. The acquired data allows gait analysis from guided-path tomography images or by machine learning. We demonstrate weight estimation with accuracy better than $1 \%$ over a range suitable for deployment in pig farms.
\end{abstract}

Keywords-distributed sensing, hostile environment, sensor enclosure, animal weight estimation, big data, IoT.

\section{INTRODUCTION}

Commercial livestock farming is a global enterprise on a scale that dwarfs most industries. The carcass value of yearly meat production is estimated at around 1 trillion USD. This immense size reflects the steady increase in meat consumption as developing nations become more dependent on animal products as a source of protein. As an example, meat consumption in BRICS countries ( $40 \%$ of world population and $25 \%$ of world GDP) rose by 6.3 percent a year in the last decade, and is expected to rise by another 2.5 percent a year until 2022 . Over and above this, there are over 270 million dairy cattle in the world, a number increasing steadily at around $1 \%$ per annum. This increase in turn creates a circumstance where ensuring the sustainability of livestock production will require, in addition to further progress in chemical and biotechnology, the wide introduction of sensors, communications and data management to improve the efficiency of production systems and complete farming units. Furthermore, the incorporation into nascent IoT structures will enable automation and information exchange across the food industry supply chain, along the concepts of Industry 4.0

The key measure of sustainability for commercial livestock performance is feed conversion ratio (FCR), or unit mass of feed required to gain a unit mass of animal. Lower FCR by a more efficient conversion indicates improved animal performance and welfare as well as reduced impact on the environment. The drive towards reduced FCR motivates farmers to monitor better the performance and understand the development of their animals.
A basic and crucial step towards this goal is for a farmer to weigh a sizeable proportion of their animals regularly.

Thus, the motivation for this work is that if farm animals could be weighed individually, precision feeding could be used to ensure accurate diet provision according to the individual animal's needs. A versatile weighing system, able to interact with farm management computing systems, has the potential to integrate with precision feeding strategies and improve FCR's across the whole range of livestock production. The potential to integrate that information beyond the farm, upstream and downstream the supply chain, is a major prerequisite for the connected farms of the future.

Current systems for weighing animals are often labour intensive and extract no information other than the total weight of the animal. A system that can weigh animals as they walk through an area of a dairy farm or even installed within a pig pen would offer significant advantages to farmers. Detecting changes in gait within the dairy industry would have the potential to identify e.g. early signs of lameness. Within the UK dairy herd, lameness costs the average dairy farmer around $£ 5000$ per year; however this varies between $£ 1500$ for the best herds and around $£ 7500$ for the worst herds, with lameness costing the UK dairy industry as a whole around $£ 50 \mathrm{M} /$ year. A cost effective method for identifying lameness early would make it possible to treat animals before an infection takes hold significantly reducing this cost.

The novelty in our work is that we demonstrate a floor sensor system for harsh environments, which is competitive in performance to mechanical weighing systems and can be deployed inconspicuously in multiples to cover the whole farm population without disturbing their daily routine, thus contributing to animal welfare. Systems for mass animal gait monitoring on the farm floor level are practically non-existent. The two variants of the system, for weight and gait, address different aspects of livestock farming, but are based on the same sensing principle.

\section{Sensing Methodology}

The reported floor sensor pilot system is based on Guided -path tomography (GPT), previously introduced [1] and tested [2] for human gait applications. It has been demonstrated that affordable GPT systems can stream human footprint data, capable of imaging the spatial distribution of the ground- 
reaction force (GRF), as well as the evolution of the footfall in the temporal domain. The sensor layer consists of parallel sets of plastic optical fiber (POF) sensor elements, manufactured inhouse from PGR-FB1000 step-index POF (Toray Industries, Inc.) of diameter $1 \mathrm{~mm}, 0.98 \mathrm{~mm}$ core of high-purity polymethyl-methacrylate and cladding of special fluorinated polymer. The POF is terminated at both ends with an optical source and a detector, respectively. The parallel sets (tomographic 'projections') are organized in several groups pointing along a small number of different equidistant angles, to provide adequate spatial sampling. An example case of 3 angles at $120^{\circ}$ is shown in Fig. 1. The depth and radius of POF deformation determines the optical transmission modulation effected by the GRF. Since the POF sensor conforms mechanically to the substrate on which it is placed, the sensor signal depends on the mechanical properties of the substrate, supporting the sensor layer. For human gait monitoring the typical substrate is a commercially available carpet underlay placed on a non-deformable surface; however, the technology is in principle scalable to map weights outside the typical range for humans, as previously demonstrated by weighing lowermass household objects [3].

Multiple small mats designed for individual weighing events with local physical mapping could be a cost effective solution to large stock density scenarios. Such mats can be used as autonomous sensors, providing a sample of the total area, or be combined to yield information over a larger scale. Consideration of extracting a population-averaged weight is possible for large stock densities by restricting the mat size to ensure data capture from a single animal.

\section{GENERAL SENSOR DESIGN AND TESTING}

Substrate materials were selected that were suitable for weighing medium farm animals such as pigs (100 to $500 \mathrm{~kg}$ total weight, corresponding to 10 to $50 \mathrm{~N} / \mathrm{cm}^{2}$ for a typical contact area). Larger and heavier animals, such as cattle, were considered a lower priority because the substantial deviation from existing GPT facilities and design. Our main pilot design "P-Mat" used a sensor head with a periodic parallel sets POF sensors arranged into 3 projections (defined as a group of parallel POF sensors directed at a chosen angle to enable tomographic sensing). The complete P-Mat consisted of a material "sandwich" of 7 layers (Shore 20 to 85) and individual thickness of 2 to $3 \mathrm{~mm}$, shown in Fig. 1 at an intermediate stage of manufacture, was tested in two experiments:

1) A hydraulic press was used to exert forces between 0 and $150 \mathrm{~N}$ (typically around $75 \mathrm{~N}$ ) onto an MDF model of a pig's hoof, with contact area $24 \mathrm{~cm}^{2}$, formed from a plaster cast. The MDF model was placed at various locations on the P-Mat and the complete set of signals was acquired. The observed linearity of the POF sensors in the range 50 to $100 \mathrm{~kg}$ is indicated by the high coefficient of determination, $\mathrm{R}^{2}=0.97$.

2) The P-Mat was placed on top of a calibrated commercial force plate (Kessler) with human volunteers both standing and walking. The comparison of the data from the two sensors, PMat and the Kessler force plate, showed a correlation coefficient of 0.997 . An additional test was performed with a $75 \mathrm{~kg}$ human repeatedly stepping on the MDF hoof model positioned at a

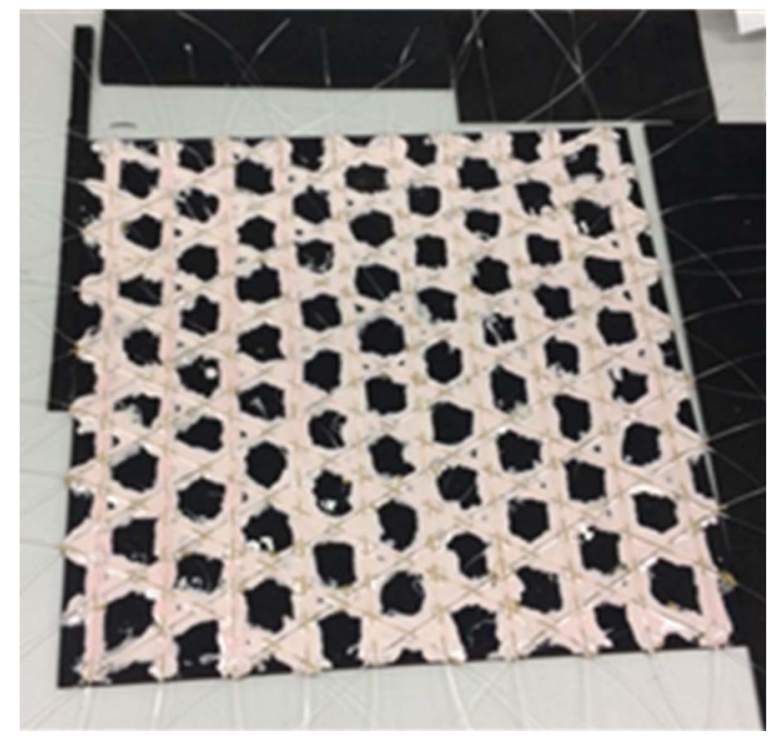

Fig.1. A P-Mat, at an intermediate stage of manufacture, showing the unconnected fibres arranged in a periodic structure. The fiber-optic grid is prepared to be placed together with the bespoke electronics into an enclosure designed to survive the farm environment without compromising performance.

single location on the mat, with and without a $0.560 \mathrm{~kg}$ on his body (see Fig. 2). Although imperfect, in terms of stability when stepping on a small size object and maintaining balance, this trial aimed at a direct answer to the question whether an accuracy better than $1 \%$ is achievable, considering the noise floor.

\section{QUANTITATIVE WEIGHT ESTIMATION FROM SINGLE PROJECTION}

Quantitative weight estimation was undertaken using only a single set of parallel POF sensors, at a $60^{\circ}$ angle to the pig's facing direction. A dummy second layer of identical unconnected POFs was incorporated underneath the active

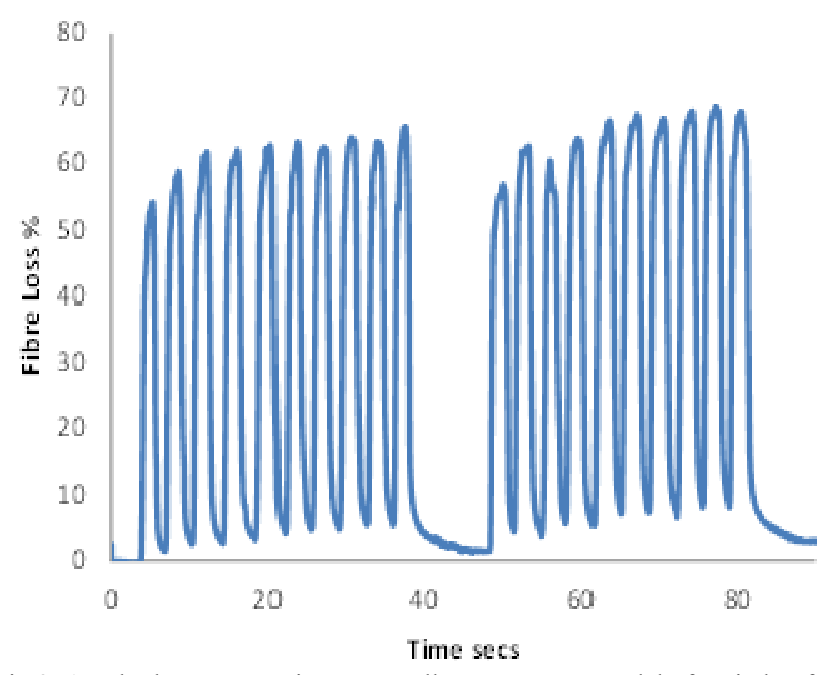

Fig 2. A $75 \mathrm{~kg}$ human stepping repeatedly on an MDF model of a pig hoof placed on the P-Mat, with ( 0 to $40 \mathrm{~s}$ ) and without (45 to $85 \mathrm{~s})$ a $0.560 \mathrm{~kg}$ eight on his body ( $<1 \%$ of body weight). The means of the two groups of 10 measurements are distinguishable well above the noise floor. 
sensor layer to reproduce realistic mechanical interaction between separate POF projections. The measurement system comprised a set of 22 POF sensors (1.05mm OD End Bright POF, terminated on both ends with OVL-3328 LEDs and TEFD4300 photodiodes) with subsequent current to voltage conversion (MIC6211). The ADC and data processing, as well as the system control functions were implemented on a ATM2560 Arduino Mega board. The digitised values were corrected for op-amp output in dark and displayed in $\mathrm{kg}$ using calibration data. The data acquisition rate was $50 \mathrm{fps}$, although rates above $30 \mathrm{fps}$ was not observed to improve the performance.

The corresponding farm-floor scenario for this experiment was weighing pigs at a relatively stationary position, e.g. while drinking water from a standard pig pen fountain. Realistic pig hoof models were used for all 4 feet of pigs at an age corresponding to weight around $100 \mathrm{~kg}$. Although in this scenario and weight bracket the position of the four hoofs is fairly well determined, the spacing between the front and back hoofs was modified to allow a single human of similar weight to step on the two pairs of hoofs simultaneously. Problems with maintaining balance, were mitigated by multiple takes of the measurement frame, as well as a large number of measurements while in position.

A typical time trace of the measurement frame is shown in Fig. 3. The observed signal-to-noise allows the estimation of the weight, after initial calibration, with accuracy better than $1 \%$. Fig. 4 shows the hoof positions and the display of the calibrated weight. The issue of the number of POF sensors contributing to the measurement frame was addressed in order to ensure that the modified front and back hoof separation was still representative of the intended scenario. Indeed, with the realistic hoof position, the distance between the parallel POF sensors and the angle at which they point ensure that each sensor is engaged by only one hoof, if any. The same applies to the realistic separation of the front and back hoofs.

In conclusion, pilot work has indicated the possibility to deploy affordable, low maintenance, low profile, permanentlyon sensor mats in pig farms with the aim to monitor the gait and weight of pigs. The first aim is relevant e.g. for monitoring of

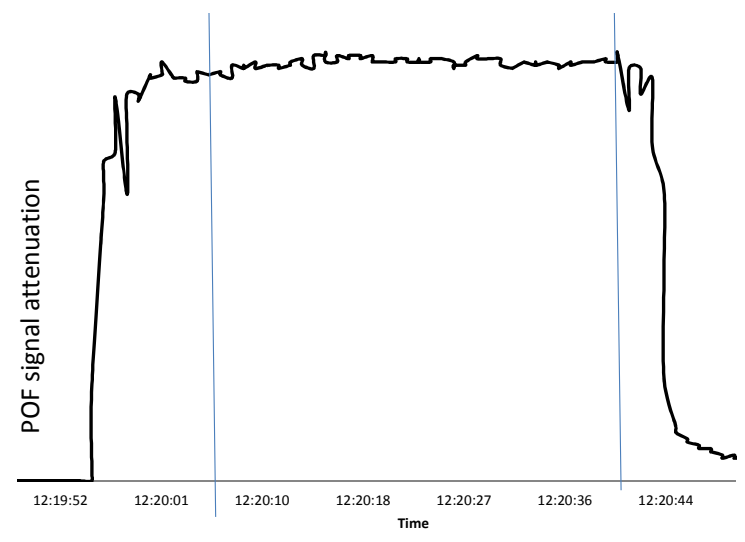

Fig.3. Human stepping on two feet (left foot on the left pair of hoofs and ight foot on the right pair of hoofs, see also Fig.4) for about 1 minute. The rertical lines show the time interval over which collected data can be used for reliable weight estimation.

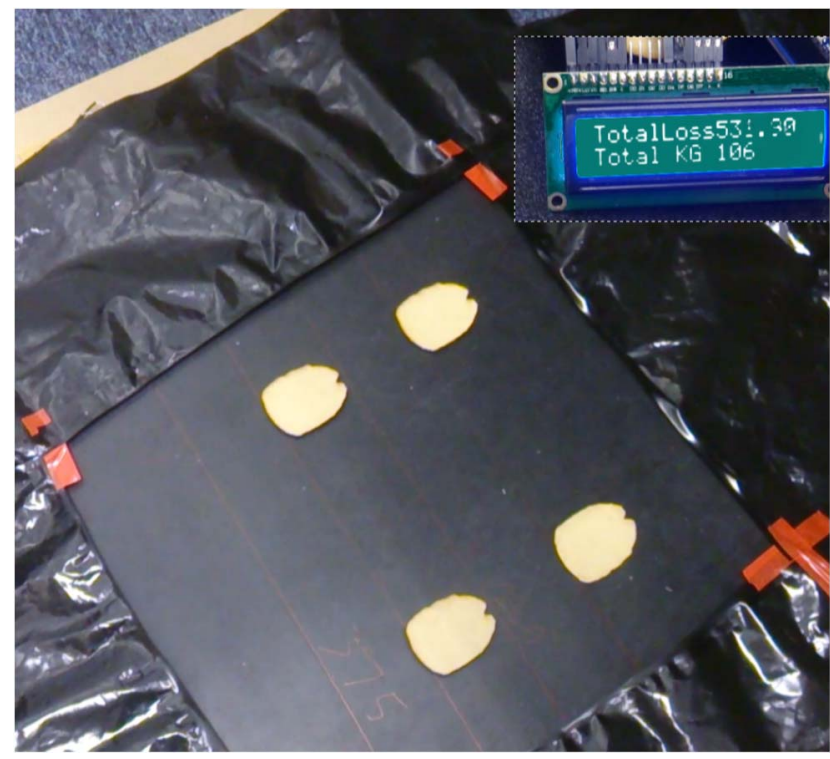

Fig 4. A $0.25 \mathrm{~m}^{2} \mathrm{P}$-Mat for weight estimation experiments, showing the position if the model hoofs (the distance between front and back hoofs is drastically reduced to allow a single human stepping with both legs). The periphery is masked to limit ambient light access.

pregnant sows, as such information is valuable for early detection of lameness and for planning their next gestation period. For this task, sensor mats can be positioned in locations where animal traffic is managed to allow gathering of data from a single sow at a time. The second task is optimal to address at drinking fountain locations. Both tasks are demanding in terms of the necessity to operate in hostile conditions - mechanical stress, aggressive liquids, etc. This requirement can be met by deploying rubberised mat sensor heads (see material "sandwich" in section III) containing zero-maintenance encapsulated electronics, connected though a single umbilical for power and data communications, to a networked node for contributing to the local general farm management, as well as the upstream and downstream food industry supply chains, including future IoT infrastructures.

\section{ACKNOWLEDGMENT}

Research Council support (EPSRC/BBSRC, UK) as well as industry (AB Agri) is gratefully acknowledged. We wish to acknowledge the Biomechanics Lab at the University of Manchester, School of MACE for access to the floor-embedded force plate, as well as the volunteers who generously made themselves available for the tests.

\section{REFERENCES}

[1] K. B. Ozanyan, S. Garcia Castillo, and F. P. Ortiz, "Guided-path tomography sensors for nonplanar mapping," Sensors Journal, IEEE, vol. 5, no. 2, pp. 167-174, 2005.

[2] J. Cantoral-Ceballos, N. Nurgiyatna, P. Wright, J. Vaughan, C. BrownWilson, P. J. Scully, K. B. Ozanyan et al., "Intelligent carpet system, based on photonic guided-path tomography, for gait and balance monitoring in home environments," Sensors Journal, IEEE, vol. 15, no. 1, pp. 279-289, 2015

[3] O. Costilla-Reyes, P. Scully and K.B. Ozanyan, " Temporal pattern recognition in gait activities recorded with a footprint imaging sensor system," Sensors Journal, IEEE, vol. 16, no. 24, pp. 8815--8822, 2016. 